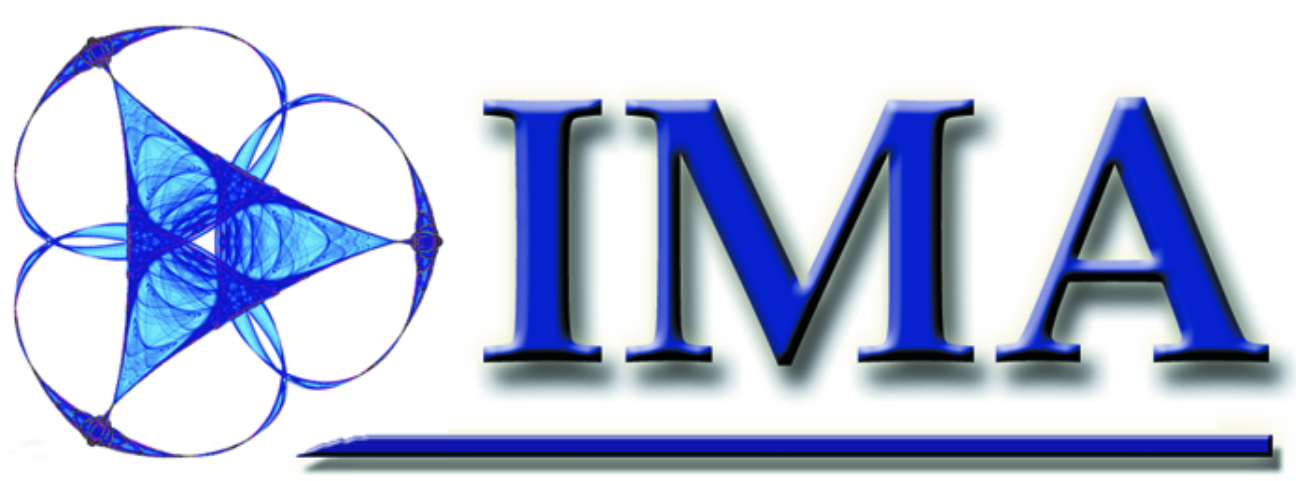

Institute for Mathematics and its Applications

\title{
COMPATIBLE SPATIAL DISCRETIZATIONS FOR PARTIAL DIFFERENTIAL EQUATIONS
}

An IMA Hot Topics Workshop

May 11-15, 2004

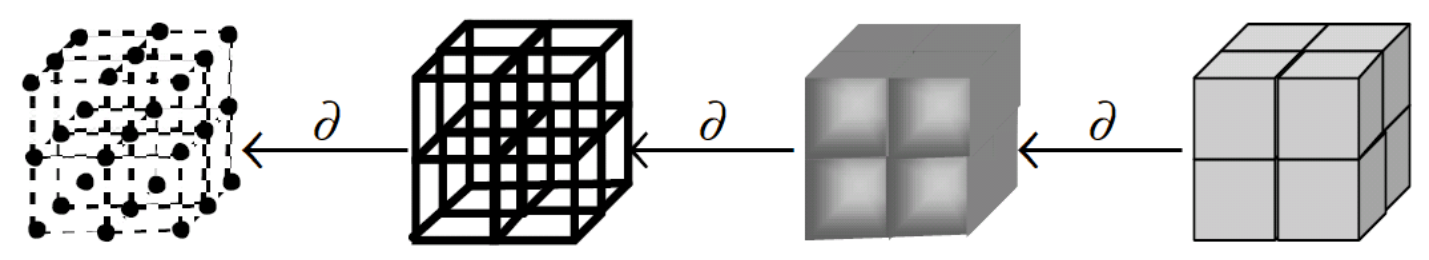


Cover image from workshop presentation of Pavel Bochev. 


\section{CONTEnTs}

Introduction $\quad 1$

Participants

PRESENTATIONS $\quad 5$

Ivar Aavatsmark: MPFA Discretization on Quadrilateral Grids 5

Peter Arbenz: Eigenvalue Solvers for Electromagnetic Fields in Cavities (poster) 7

Douglas N. Arnold: Differential Complexes and Stability of Finite Element Methods 8

Timothy J. Barth: Entropy Stable FEM Discretization of First-Order Systems of Conservation Laws 9

Martin Berggren: A Vertex-Centered Dual Discontinuous Galerkin Method (poster) 10

Pavel Bochev: Variational and Geometric Aspects of Compatible Discretizations 11

Daniele Boffi: Compatible Discretizations for Eigenvalue Problems 12

Alain Bossavit: Computational Electromagnetism and Whitney Forms 13

Panagiotis Chatzipantelidis: A Finite Volume Element Method for a Nonlinear Elliptic Problem 14

Snorre H. Christiansen: Div-curl Lemma for Edge Elements (poster) 15

Leszek Demkowicz: De Rham Diagram for Projection-Based Interpolation. 3D Optimal p- and hp-Error Estimates (poster) 16

Yalchin Efendiev: Numerical Homogenization of Nonlinear Partial Differential Equations and its Applications

17

Jürgen Geiser: Mixed Discretisation Methods for Discontinuous Galerkin Method with Analytical $\begin{array}{ll}\text { Test-Functions (poster) } & 18\end{array}$

Ralf Hiptmair: Higher Order Whitney Forms 19

Anil N. Hirani: Discrete Exterior Calculus and its Applications in Mechanics and Computer Science (poster) 20

Thomas J.R. Hughes: "Consider a Spherical Cow" - Conservation of Geometry in Analysis: Implications for Computational Methods in Engineering

P. Robert Kotiuga: Intuitive vs. Computable Topological Aspects of Computational Electromagnetics (poster) 22

Melvin Leok: Discrete Connections on Principal Bundles (poster) 23

Konstantin Lipnikov: New Mimetic Discretizations of Diffusion-Type Problems on Polygonal Meshes (poster) 24

Elizabeth L. Mansfield: Towards a Variational Complex of the Finite Element Method 25

Ilia D. Mishev: Why Mixed Finite Elements are not used in the Petroleum Industry and what can we do about it? (poster) 26

Jim E. Morel: Asymptotic-Preserving Discretization Schemes 27

J. David Moulton: Mimetic Preconditioners for Mixed Discretizations of the Diffusion Equation (poster) 28

Roy Nicolaides: Compatible Discretizations, Covolume Algorithms and Differential Forms 29

Blair Perot: Compatible Reconstructions of Vectors and their Application to the Navier-Stokes Equations

Ilaria Perugia: Discontinuous Galerkin Methods for Maxwell's Equations in Frequency-Domain (poster)

Robert N. Rieben: High Order Symplectic Integration Methods for Finite Element Solutions to Time Dependent Maxwell Equations (poster) 32

Beatrice Riviere: Two-Phase Flow Modeling (poster) 33

Allen C. Robinson: Compatible Discretizations in Lagrangian/Eulerian Resistive MHD Modeling for Z-pinch Applications (poster) 
Thomas F. Russell: Relationships Among Some Locally Conservative Discretization Methods Which Handle Discontinuous Anisotropic Coefficients on Deformed Grids

Rolf Schuhmann: Consistent Material Operators for Geometrical Discretization Methods on Generalized Grids (poster)

Mikhail Shashkov: Mimetic Finite Difference Methods for Partial Differential Equations and Discrete Vector and Tensor Analysis

Rajen Kumar Sinha: Finite Volume Element Methods for Parabolic Integro-Differential Equation with Nonsmooth Initial Data (poster) 38

Eitan Tadmor: Entropy Stability Theory For Difference Approximations of Quasilinear Problems 39

Jean-Marie Thomas: Finite Element Methods for Non-elliptic but Coercive Problems (poster) 40

Jukka Tuomela: Formal Theory of PDEs and simulation of Fluid Flows (poster)

Mary Fanett Wheeler: Coupling of MFE or Mimetic Finite Differences with Discontinuous Galerkin for Poro-Elasticity

Daniel A. White: Development and Application of Compatible Discretizations of Maxwell's Equations 43

Ragnar Winther: From the de Rham Sequence to Mixed Elasticity 


\section{Introduction}

From May 11-15, 2004, the Insitute for Mathematics and its Applications held a hot topics workshop on Compatible Spatial Discretizations for Partial Differential Equations.

The numerical solution of partial differential equations (PDE) is a fundamental task in science and engineering. The goal of the workshop was to bring together a spectrum of scientists at the forefront of the research in the numerical solution of PDEs to discuss compatible spatial discretizations. We define compatible spatial discretizations as those that inherit or mimic fundamental properties of the PDE such as topology, conservation, symmetries, and positivity structures and maximum principles. A wide variety of discretization methods applied across a wide range of scientific and engineering applications have been designed to or found to inherit or mimic instrinsic spatial structure and reproduce fundamental properties of the solution of the continuous PDE model at the finite dimensional level. A profusion of such methods and concepts relevant to understanding them have been developed and explored: mixed finite element methods, mimetic finite differences, support operator methods, control volume methods, discrete differential forms, Whitney forms, conservative differencing, discrete Hodge operators, discrete Helmholtz decomposition, finite integration techniques, staggered grid and dual grid methods, etc. This workshop seeks to foster communication among the diverse groups of researchers designing, applying, and studying such methods as well as researchers involved in practical solution of large scale problems that may benefit from advancements in such discretizations; to help elucidate the relations between the different methods and concepts; and to generally advance our understanding in the area of compatible spatial discretization methods for PDE.

Particular points of emphasis included:

- Identification of intrinsic properties of PDE models that are critical for the fidelity of numerical simulations.

- Identification and design of compatible spatial discretizations of PDEs, their classification, analysis, and relations.

- Relationships between different compatible spatial discretization methods and concepts which have been developed;

- Impact of compatible spatial discretizations upon physical fidelity, verification and validation of simulations, especially in large-scale, multiphysics settings.

- How solvers address the demands placed upon them by compatible spatial discretizations.

\section{Organizers}

Douglas N. Arnold. IMA, University of Minnesota

Pavel Bochev. Computational Mathematics and Algorithms Department, Sandia National Laboratories

Rich Lehoucq. Computational Mathematics and Algorithms Department, Sandia National Laboratories

Roy Nicolaides. Department of Mathematical Sciences, Carnegie-Mellon University.

Mikhail Shashkov. MS-B284, Group T-7, Theoretical Division, Los Alamos National Laboratory 


\section{Participants}

Aavatsmark, Ivar. University of Bergen

Adams, Scot. University of Minnesota

Arbenz, Peter. ETH Zentrum

Arnold, Douglas N. University of Minnesota

Aronson, Donald G. University of Minnesota

Awanou, Gerard. University of Minnesota

Bank, Randolph E. University of California - San Diego

Barth, Timothy J. NASA Ames Research Center

Berggren, Martin. Uppsala University

Bochev, Pavel. Sandia National Laboratories

Boffi, Daniele. Universita di Pavia

Bossavit, Alain. Laboratoire de Génie Electrique de Paris

Brezhneva, Olga. University of Minnesota

Brezzi, Franco. I. A. N. del C. N. R.

Cai, Zhiqiang. Purdue University

Castillo, Jose E. San Diego State University

Chatzipantelidis, Panagiotis. Texas A \& M University

Christiansen, Snorre H. Centre of Mathematics for Applications

Christon, Mark. Sandia National Laboratories

Cockburn, Bernardo. University of Minnesota

Crone, Bob. Seagate Technology

Demkowicz, Leszek. University of Texas - Austin

Efendiev, Yalchin. Texas A \& M University

Falk, Richard. Rutgers

Geiser, Juergen. Texas A \& M University

Gundel, Anne. Humboldt University Berlin

Hamdan, Hazem. University of Minnesota

He, Bo. Ohio State University

Hesthaven, Jan S. Brown University

Hetmaniuk, Ulrich. Sandia National Laboratories

Hiptmair, Ralf. ETH Zentrum

Hirani, Anil N. California Institute of Technology

Holst, Michael J. University of California - San Diego

Hoppe, Ronald. University of Houston

Houston, Paul. University of Leicester

Hughes, Thomas J.R. University of Texas - Austin

Hyde, E. Mckay. University of Minnesota

Ju, Lili. University of Minnesota

Juanes, Ruben. Stanford University

Kim, Hye-Ryoung. Seoul National University (BK21)

Koning, Joseph M. Lawrence Livermore National Laboratories

Kotiuga, Robert P. Boston University

Kurtz, Thomas G. University of Wisconsin

Kuznetsov, Yuri. University of Houston

Lazarov, Raytcho. Texas A \& M University

Lehoucq, Richard. Sandia National Laboratories

Leok, Melvin. California Institute of Technology

Lipnikov, Konstantin. Los Alamos National Laboratory

Luskin, Mitchell. University of Minnesota

Maniatty, Antoinette. Rensselaer Polytechnic Institute 
Mansfield, Elizabeth L. University of Kent at Canterbury

Marini, Donatella. University of Pavia

Mishev, Ilya D.. ExxonMobil

Mitchell, Julie C. University of Wisconsin

Morel, Jim. Los Alamos National Laboratory

Moulton, J. David. Los Alamos National Laboratory

Nedelec, Jean-Claude. Ecole Polytechnique

Nicolaides, Roy. Carnegie Mellon University

Pebay, Philippe P. Sandia National Laboratories

Perot, Blair. University of Massachusetts

Perugia, Ilaria. Universita di Pavia

Ratner, Edward. KLA-Tencor

Reitich, Fernando. University of Minnesota

Remacle, Jean-Francois. Rensselaer Polytechnic Institute

Rieben, Robert N. Lawrence Livermore National Laboratories

Riviere, Beatrice M. University of Pittsburgh

Robinson, Allen. Sandia National Laboratories

Russell, Thomas F. National Science Foundation

Santosa, Fadil. University of Minnesota

Schuhmann, Rolf. Darmstadt University of Technology

Shashkov, Mikhail. Los Alamos National Laboratory

Shih, Shagi-Di. University of Wyoming

Sinha, Rajen Kumar. Texas A \& M University

Steinberg, Stanly. University of New Mexico

Stojanovic, Srdjan. University of Cincinnati

Tadmor, Eitan. University of Maryland

Teixeira, Fernando Lisboa. Ohio State University

Thomas, Jean-Marie. University of Pau and the Countries of Adour

Trapp, Kathryn A. Carnegie Mellon University

Tuomela, Jukka. University of Joensuu

Vassilevski, Panayot. Lawrence Livermore National Laboratories

Wang, Jing. University of Minnesota

Warburton, Tim. University of New Mexico

Wheeler, Mary Fanett. University of Texas - Austin

White, Daniel A. Lawrence Livermore National Laboratories

Winther, Ragnar. Universitetet of Oslo

$\mathbf{X u}$, Jinchao. Pennsylvania State University

Yotov, Ivan. University of Pittsburgh

Zhao, Jun. University of Minnesota 


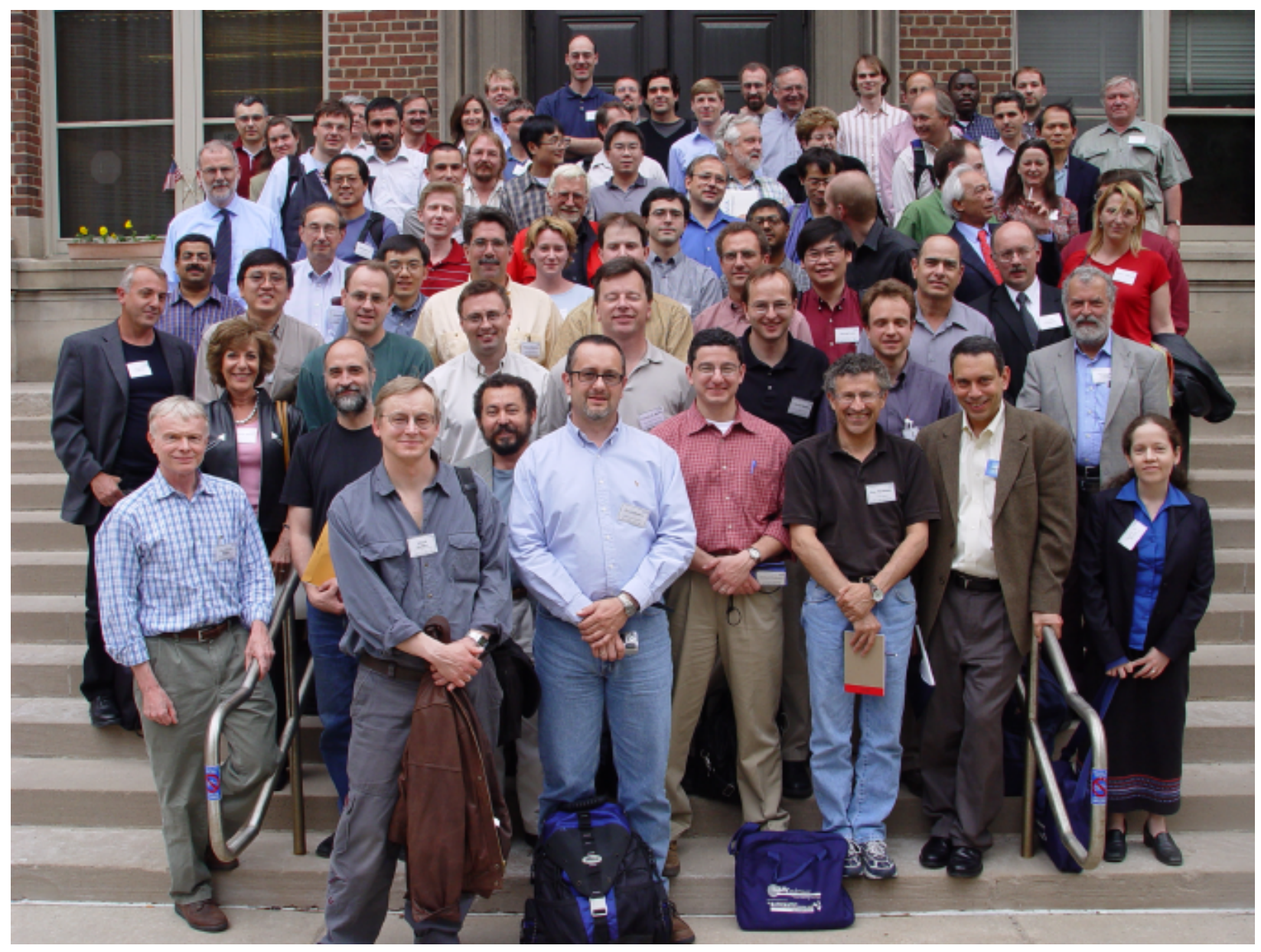




\section{PRESENTATION ABSTRACTS}

Materials from presentations are available at www.ima.umn.edu/complex/spring/discretization.html 


\title{
MPFA Discretization on Quadrilateral Grids
}

\author{
Ivar Aavatsmark \\ Senter for integrert petroleumsforskning, Universitetet i Bergen
}

\begin{abstract}
The presentation will give an overview of the MPFA methods and its applications in multiphase flow in porous media. Recent progress on the theoretical side will be covered: Convergence proof for one MPFA method, monotonicity properties in the homogeneous case, new variants of the MPFA method, convergence properties when the Edwards splitting is used as preconditioner.
\end{abstract}




\title{
Eigenvalue Solvers for Electromagnetic Fields in Cavities (poster)
}

\author{
Peter Arbenz \\ Institute of Computational Science, Swiss Federal Institute of Technology
}

\begin{abstract}
Joint work with M. Becka, Institute of Computational Science, ETH Zurich R. Geus, PaulScherrer Institute, Villingen U. Hetmaniuk, Sandia National Laboratories, Albuquerque.

We investigate the Jacobi-Davidson algorithm for computing a few of the smallest eigenvalues of a generalized eigenvalue problem resulting from the finite element discretization of the time-harmonic Maxwell equation. Various multilevel preconditioners are employed to improve the convergence rate and memory consumption of the eigensolver. We present sequential results of very large eigenvalue problems originating from the design of resonant cavities of particle accelerators. Furthermore we detail our approach for parallelizing our code by means of the Trilinos software framework.
\end{abstract}




\title{
Differential Complexes and Stability of Finite Element Methods
}

\author{
Douglas N. Arnold \\ IMA, University of Minnesota
}

\begin{abstract}
Many of the partial differential equations of mathematical physics are related to differential complexes which determine their structure and well-posedness. Many successful finite element discretizations of these problems can best be understood as arising from piecewise polynomial subcomplexes. The stability of these methods is obtainted by relating the discrete subcomplex to the continuous differential complex via a commuting diagram. The best known case is the de Rham complex, which underlies both electromagnetic and diffusion problems. In this case there are a large number of possible piecewise polynomial subcomplexes of each order. These can be presented systematically using the Koszul complex. The elasticity equations are related to another differential complex which can be related to the de Rham complex through a subtle homological construction. This has lead to recent progress in the design of stable mixed finite elements for elasticity in two and three dimensions.
\end{abstract}




\title{
Entropy Stable FEM Discretization of First-Order Systems of Conservation Laws
}

\author{
Timothy J. Barth \\ NASA Ames Research Center
}

\begin{abstract}
We consider the discontinuous Galerkin (DG) finite element discretization [RH73, CLS89, CHS90, Coc99] of first-order systems of conservation laws such as those occurring in hydrodynamics. Stability of the discontinuous Galerkin method depends critically on the choice of the numerical flux and any other stabilization added in element interiors or on element interfaces. In this lecture, we give a self-contained review of global and elementwise local entropy analysis for the DG method and how the analysis is used to design stable numerical fluxes. Consequently, using these discretizations, global and elementwise local entropy inequalities are formally obtained. For example, if $U$ denotes the convex entropy, then a formal space-time global entropy inequality for a spatial domain is obtained

$$
\int_{\Omega} U\left(T_{0}+n \Delta t\right) d x \leq \int_{\Omega} U\left(T_{0}\right) d x+\text { boundary, } \quad n=0,1,2, \ldots
$$

for a prescribed time slab increment $t$. We then expand the discussion to include a first-order system of conservation laws with solenoidal constraint such as those occurring in compressible magnetohydrodynamics (MHD). The objective is to again show how entropy analysis plays an invaluable role in designing numerical fluxes and stabilization terms. Unlike standard hydrodynamics, the entropy analysis for MHD reveals the subtle role of the solenoidal constraint in obtaining global and elementwise local entropy stability

- strong or weak (penalty) satisfaction of $\operatorname{div} B=0$ in element interiors

- strong or weak (penalty) satisfaction of $[B \cdot n]=0$ on element interfaces.

Although one could satisfy $[B \cdot n]=0$ strongly (see for example Brezzi-Douglas- Marini (1985)), this would add considerable complexity to the discontinuous Galerkin method and would destroy the simple block diagonal mass matrix structure in the DG method. Alternatively, one can use the entropy analysis to design a $[B \cdot n]=0$ penalty term with magnitude dictated by the entropy analysis so that global and elementwise local entropy inequalities are obtained and the simple mass matrix structure retained. Finally, we consider the generalization of these procedures to other systems of conservations laws with constraints. Throughout the lecture, numerical results of interest are given to verify the analysis.
\end{abstract}

[Bar99] T.J. Barth. Simplified discontinuous Galerkin methods for systems of conservation laws with convex extension. In Cockburn, Karniadakis, and Shu, editors, Discontinuous Galerkin Methods, volume 11 of Lecture Notes in Computational Science and Engineering. Springer-Verlag, Heidelberg, 1999.

[CHS90] B. Cockburn, S. Hou, and C.W. Shu. TVB Runge-Kutta local projection discontinuous Galerkin finite element method for conservation laws IV: The multidimensional case. Math. Comp., 54:545581, 1990.

[CLS89] B. Cockburn, S.Y. Lin, and C.W. Shu. TVB Runge-Kutta local projection discontinuous Galerkin finite element method for conservation laws III: One dimensional systems. J. Comp. Phys., 84:90113, 1989.

[Coc99] B. Cockburn. Discontinuous Galerkin methods for convection-dominated problems. In Barth and Deconinck, editors, High-Order Discretization Methods in Computational Physics, volume 9 of Lecture Notes in Computational Science and Engineering, pages 69224. Springer-Verlag, Heidelberg, 1999.

[God72] S. K. Godunov. The symmetric form of magnetohydrodynamics equation. Num. Meth. Mech. Cont. Media, $1: 2634,1972$.

[RH73] W. H. Reed and T. R. Hill. Triangular mesh methods for the neutron transport equation. Technical Report LA-UR-73-479, Los Alamos National Laboratory, Los Alamos, New Mexico, 1973. 


\title{
A Vertex-Centered Dual Discontinuous Galerkin Method (poster)
}

\author{
Martin Berggren \\ Department of Scientific Computing, Uppsala University
}

\begin{abstract}
I will present a new discontinuous Galerkin method for discretizing partial differential equations with a dominating hyperbolic character. At lowest order, the method reduces to a vertex-centered finite-volume method with control volumes based on a dual mesh, and the method can be implemented using an edge-based data structure. Preliminary tests on a model linear hyperbolic equation in 2D indicate a favorable qualitative behavior for nonsmooth solutions, and an optimal convergence rate for smooth solutions when using locally piecewise-linear approximations.
\end{abstract}




\title{
Variational and Geometric Aspects of Compatible Discretizations
}

\author{
Pavel Bochev \\ Computational Mathematics and Algorithms and Computer Science Research Institute, Sandia \\ National Laboratories
}

\begin{abstract}
PDE models arise in virtually all fields of science and engineering. Their compatible discretizations are finite dimensional models of the physical process that are stable and provide accurate and physically meaningful solutions.

Variational principles take advantage of the intrinsic connections between the structure of many PDEs and optimization problems to identify their compatible discretizations.

Differential complexes provide another tool to encode the structure of a PDE. Differential forms represent global quantities rather than fields, and provide a model for the way we observe the physical process. The idea that differential forms can and should be used to develop compatible (mimetic) discretizations started to permeate computational sciences approximately two decades ago and led to fundamental advances in computational electromagnetics.

Since then, geometrical approaches to discretization have enjoyed a steady and ever increasing interest and appreciation in computational sciences. The goal of this lecture is two-fold. I will show how variational and geometric techniques can complement each other in the quest for accurate and stable discretizations by providing tools for the analysis and the design of compatible models. In doing so I will retrace the key steps that have led to our modern understanding of these connections. To illustrate, as well as to compare and contrast variational identifications of compatibility, I will use the Kelvin and the Dirichlet principles. Factorization diagrams will reveal the geometrical structure of the problem and form the basis for the design of compatible discretizations. Then, with the help of the grid decomposition property and the commuting diagram property I will show the fundamental links between the two approaches.
\end{abstract}

I will conclude with examples of alternative discrete models that circumvent the rigid structural constraints imposed by compatibility and talk about the advantages and the perils associated with their use. 


\title{
Compatible Discretizations for Eigenvalue Problems
}

\author{
Daniele Boffi \\ Dipartimento di Matematica Universit di Pavia
}

\begin{abstract}
We start with a short review of standard Galerkin approximation of variationally posed eigenvalue problems, where we mainly consider the case of elliptic problems associated with a compact resolvent. Our basic example is the Laplace/Poisson eigenvalue problem. The main result, in this case, can be summarized by the claim that any choice of discrete space sequence, which provides a convergent scheme for the source problem, automatically performs well when applied to the corresponding eigenvalue problem. The main discussion in this talk focuses on the approximation of eigenvalue problems in mixed form. Using again the basic example of the Laplace/Poisson eigenvalue problem, we show that, when using a mixed method for its discretization, the picture is somewhat different from the previous case. The main (counter) example is given by a choice of discrete space sequences such that the classical Brezzi's conditions are satisfied (whence the source problem is correctly approximated) but, when the eigenvalue problem is considered, several spurious eigenmodes pollute the discrete spectrum. This surprising behavior is proved theoretically and numerically demonstrated. We then review the theory of the discretization of eigenvalue problems in mixed form (joint work with F. Brezzi and L. Gastaldi), where the compatibilities between the discrete space sequences for the good approximation of eigenpairs are made clear. Necessary and sufficient conditions are given for several general cases of interest. If time permits, some consequences of the theory can be presented. One application of the theory is the approximation of interior Maxwell's eigenvalue (leading in particular to the discretization of time-harmonic Maxwell's system). The results can be suitably modified to include the approximation of band gaps for photonic crystals (joint work with M. Conforti and L. Gastaldi). Another important consequence concerns the approximation to evolution problems in mixed form (joint work with L. Gastaldi).
\end{abstract}




\title{
Computational Electromagnetism and Whitney Forms
}

\author{
Alain Bossavit \\ Laboratoire de Gnie Electrique de Paris
}

\begin{abstract}
This talk will give an overview of the use of Whitney forms in electromagnetism, from 1980 to now, and of desirable developments.

Edge elements (not to be known by that name until about 1986) allowed to solve eddy current problems in dimension 3 , a notorious conundrum during the 70's. Within ten years, edge element discretization, Galerkin style, became the established method in computational electromagnetism, (CEM) while efforts to answer the question "why edge elements?" slowly fostered familiarity with Whitney forms and cohomology in the CEM community. Once the central place of cohomology and commutative diagrams was acknowledged, ideas about "mimetic" discretizations, equivalent network methods, etc., could develop in the 90's, and the order of the day, now, is to found an appropriate "discrete exterior calculus" - which holds promises far beyond electromagnetics. Lots of questions remain, however: Convergence issues, Whitney forms on non-simplicial meshes, in particular, will be addressed. (Some new results about the geometric interpretation of degrees of freedom for higher-degree Whitney forms will be presented.) As for the future, the demands of engineering about coupled problems (such as magneto-elasticity, MHD, etc.) will promote a better understanding of the differential-geometric structures which underlie both electromagnetism and continuum mechanics.
\end{abstract}




\title{
A Finite Volume Element Method for a Nonlinear Elliptic Problem
}

\author{
Panagiotis Chatzipantelidis \\ Department of Mathematics, Texas A\&M University
}

\begin{abstract}
Joint work with: V. Ginting and R.D. Lazarov.
We consider a finite volume discretization of second order nonlinear elliptic boundary value problems on polygonal domains. For sufficiently small data, we show existence and uniqueness of the finite volume solution using a fixed point iteration method. We derive error estimates in $H^{1}, L^{2}$ and $L^{\infty}$ norms. In addition a Newton's method is analyzed for the approximation of the finite volume solution and numerical experiments are presented.
\end{abstract}




\title{
Div-curl Lemma for Edge Elements (poster)
}

\author{
Snorre H. Christiansen \\ Centre of Mathematics for Applications CMA, Department of Mathematics, University of Oslo
}

\begin{abstract}
A variant of Murat and Tartar's div-curl lemma is stated and proved for Nedelec's edge elements. In the Galerkin finite element setting one can expect control of $L^{2}$ norms of vectorfields and also sufficient control of their curls in $H^{1}$. But the divergence is usually just controlled trough the integral of the vectorfields against a finite dimensional space of gradients. The proposed lemma is designed to handle this case. The proof uses a uniform norm equivalence related to discrete compactness properties of vector FE spaces and a super-approximation property of scalar FE spaces.
\end{abstract}




\title{
De Rham Diagram for Projection-Based Interpolation. 3D Optimal p- and hp-Error Estimates (poster)
}

\author{
Leszek Demkowicz \\ Texas Institute for Computational Engineering Sciences, University of Texas at Austin
}

\begin{abstract}
I will present the main idea and results for the commuting de Rham diagram for polynomial spaces corresponding to 3D finite elements of variable order generalizing Nedelec tetrahedrons of the first and second type, hexahedron of the first type (Nedelec's hexahedron of the second type does not satisfy the commuting diagram property), and prisms of the first and second type.

I will discuss shortly the generalization to parametric elements, including the most popular isoparametric elements.

The optimal p-interpolation (and following hp-interpolation) estimates are obtained by comparing the interpolation errors with (commuting) projections errors. Two fundamental tools necessary for the interpolation error analysis include recent results on existence of continuous, polynomial preserving extension operators (with M. Ainsworth) and discrete Friedrichs inequalities (with J. Gopalakrishnan). The presented interpolation theory summarizes a recent work done with A. Buffa. The methodology differs considerably from earlier 2D results obtained with I. Babuska.
\end{abstract}




\title{
Numerical Homogenization of Nonlinear Partial Differential Equations and its Applications
}

\author{
Yalchin Efendiev \\ Department of Mathematics, Texas A\&M University
}

\begin{abstract}
The numerical homogenization methods presented in this talk are designed to compute homogenized solutions. In particular we are interested when the heterogeneities have random nature. I will describe numerical homogenization methods that we proposed recently and their relation to some other multiscale methods. Convergence of these methods for nonlinear parabolic equations will be discussed. Numerical examples and applications will be considered.
\end{abstract}




\title{
Mixed Discretisation Methods for Discontinuous Galerkin Method with Analytical Test-Functions (poster)
}

\author{
Jürgen Geiser \\ IWR, University of Heidelberg, Visiting professor of ISC, Texas A\&M University, College Station
}

\begin{abstract}
Joint work with: R. Lazarov and R. Ewing.
Our mathematical models describe transport and reaction processes in porous media. Based on our model equations we present a new mixed discretization methods with analytical test-functions and the error-analysis. The based convection-diffusion-reaction-equations are discretised with Discontinuous Galerkin methods in a mixed formulation (LDG-method).
\end{abstract}

We introduce the variational formulations and our adjoint problem to derive the analytical solutions for the testfunctions. The stability of the discretization methods are discussed and an abstract error-estimates is derived. We apply the abstract error-estimates for the different test-functions, e.g. polynomial- and exponential-functions and present an improved optimal order result for our new exponential test-functions.

The application for our new discretization methods are proposed. Finally we discuss our further works.

Key words: convection-diffusion-dispersion-reaction-equation, Discontinuous Galerkin method, analytical methods 


\title{
Higher Order Whitney Forms
}

\author{
Ralf Hiptmair \\ Seminar for Applied Mathematics, ETH-Zentrum
}

\begin{abstract}
Compatible finite element discretizations of second-order boundary value problems set in the function spaces $\mathrm{H1}($ ), $\mathrm{H}(\mathrm{curl}$, ), and $\mathrm{H}($ div, ) will naturally rely on discrete differential forms. Their lowest-order representatives are the well-knowns linear Lagrangian finite elements, edge elements, and face elements. However, given the interior smoothness of the solutions of many boundary value problems, approximation by local polynomials of high degree offers superior efficiency (hp-version of FEM).

The first part of the presentation will focus on the algebra behind the construction of higher order Whitney forms. The calculus of differential forms will be used to devise a unified description of discrete differential forms of any order and polynomial degree on simplicial meshes in any spatial dimension. A general formula for suitable degrees of freedom is also available. Fundamental properties of nodal interpolation can be established easily. It turns out that higher order spaces, including variants with locally varying polynomial order, emerge from the usual Whitney-forms by local augmentation.

The second part of the talk will review the concrete construction of suitable basis functions for higher order Whitney forms. Discrete 1-forms will serve as main example. Recent inventions of p-hierarchical bases with good conditioning properties will be discussed.

Time permitting, the third part of the talk will investigate p-uniform stability properties of high order Whitney forms. It will show how tools from differential geometry and discrete topology can be used to obtain a p-uniform discrete Poincar-Friedrichs inequality that plays a key role in the convergence theory for the p-version of discrete differential forms.
\end{abstract}




\title{
Discrete Exterior Calculus and its Applications in Mechanics and Computer Science (poster)
}

\author{
Anil N. Hirani \\ Control and Dynamical Systems, Caltech
}

\begin{abstract}
To solve PDEs on irregular, non-flat meshes, one can either interpolate and compute differential quantities, or define a discrete calculus without interpolation while preserving some of the structure of the smooth theory. Discrete exterior calculus (DEC) is tensor analysis on irregular, simplicial meshes and their duals, which takes the latter approach. It defines differential operators on such meshes in a coordinate independent way. With it, one can solve, for example, Laplace's equation on an arbitrary triangle mesh surface. Most numerical methods except FEM, are for flat, regular grids. While FEM involves interpolation of scalar values on irregular grids, DEC works with interpolations of values defined on points, edges, triangles etc. In addition, it provides a way to incorporate vector fields into such a framework, thus allowing for computations on moving meshes. We will describe DEC and suggest some of its applications in computer science and mechanics.
\end{abstract}




\title{
"Consider a Spherical Cow" - Conservation of Geometry in Analysis: Implications for Computational Methods in Engineering
}

\author{
Thomas J.R. Hughes \\ Institute for Computational Engineering and Sciences, University of Texas at Austin
}

\begin{abstract}
Consider the spherical cow" is the punch line of a mathematics joke. The joke itself is not so important to the subject of this work but the message is, namely, that simplifications of geometry are often made to facilitate analysis. Let us take the engineering design process as an example. There are estimated to be of the order of a million analyses a day performed in engineering design offices throughout the world. Engineering designs are encapsulated in Computer Aided Design (CAD) systems. Up to manufacturing tolerances, these systems exactly represent the geometry of designs. The analysis process begins with CAD geometry but the predominate method of analysis, finite elements, requires a different representation of geometry. This creates two problems: 1) The need to generate the geometric description suitable for the finite element method; and 2) the geometric errors that are produced in the process. The first problem, "mesh generation," is attributed to taking over 80industries such as shipbuilding, aerospace and automotive. It has become the major bottleneck in engineering analysis. The second problem is very important in certain situations, such as, for example, the buckling of thin shells, which exhibit strong geometric imperfection sensitivity. Since approximating the geometry for analysis purposes is costly, time consuming, and potentially creates significant errors, it raises the question, why do we do it? It would seem beneficial to conserve the exact CAD geometry in analysis, up to, of course, features that we definitely want to remove. This work takes the point of view that conserving geometry is an important conservation law that should be satisfied. We pursue this idea and see where it takes us. It suggests a very different analytical structure but one in which mesh generation may be dramatically simplified. Some simple computations in structural analysis are presented which indicate the ideas are viable and we argue why we feel that developing a complete mathematical convergence theory should be straightforward.
\end{abstract}




\title{
Intuitive vs. Computable Topological Aspects of Computational Electromagnetics (poster)
}

\author{
P. Robert Kotiuga \\ Department of Electrical \& Computer Engineering, Boston University
}

\begin{abstract}
Intuitive problems, such as checking if a space is contractible, are easily characterized in terms of homotopy groups but, in four or more dimensions, such a characterization is provably computationally intractable. On the other hand, cohomology theory may not be intuitive, but it does provide a formal connection between Maxwell's equations and the lumped parameters occurring in Kirchhoff's laws. Also, cohomological information is efficiently extracted from the data structures used in finite element analysis. A natural question is: Do engineers need to go beyond the linear algebra and sparse matrix techniques associated with homology calculations? It turns out that there are inverse problems involving "near force-free magnetic fields" where the conjectured characterization of the space of solutions, involves computationally intractable topological invariants. Hence, it is imperative to investigate algebraic structures found in the data structures of finite element analysis, which yield topological insights not deducible from cohomological considerations alone.
\end{abstract}

The Hurewicz map is a well-defined map taking representatives of generators of homotopy groups to their homology classes. In this sense, it provides a natural framework for comparing the intuitive but intractable with the computable but less intuitive. The presentation will develop this theme in the context of computational electromagnetics. 


\title{
Discrete Connections on Principal Bundles (poster)
}

\author{
Melvin Leok \\ Control and Dynamical Systems, California Institute of Technology
}

\begin{abstract}
Connections on principal bundles play a fundamental role in expressing the equations of motion for mechanical systems with symmetry in an intrinsic fashion. A discrete theory of connections on principal bundles is constructed by introducing the discrete analogue of the Atiyah sequence, with a connection corresponding to the choice of a splitting of the short exact sequence.

Equivalent representations of a discrete connection are considered, and an extension of the pair groupoid composition, that takes into account the principal bundle structure, is introduced. Computational issues, such as the order of approximation, are also addressed. Discrete connections provide an intrinsic method for introducing coordinates on the reduced space for discrete mechanics, and provide the necessary discrete geometry to introduce more general discrete symmetry reduction.

In addition, discrete analogues of the Levi-Civita connection, and its curvature, are introduced by using the machinery of discrete exterior calculus, and discrete connections.

This is part of a broader program to develop discrete analogues of differential geometry which are relevant to the systematic construction of computational geometric mechanics.

Joint work with Jerrold E. Marsden (Caltech) and Alan D. Weinstein (Berkeley).
\end{abstract}




\title{
New Mimetic Discretizations of Diffusion-Type Problems on Polygonal Meshes (poster)
}

\author{
Konstantin Lipnikov \\ Los Alamos National Laboratory
}

\begin{abstract}
Joint work with Yuri Kuznetsov and Mikhail Shashkov.
The determining factor for reliability, accuracy, and efficiency of simulations is accurate locally conservative discretizations. Practice experience shows that the most effective discrete approximations preserve and mimic the underlying properties of original continuum differential operators. One of such approaches, the mimetic finite difference technique based on the support-operator methodology, has been applied successfully to several applications including diffusion, electromagnetics and gas dynamics.
\end{abstract}

As mathematical modeling becomes more sophisticated, the need for discretization methods handling meshes with mixed types of elements has arisen. On this poster we present new mimetic discretizations on polygonal meshes. AMR meshes, non-matching meshes and meshes with non-convex cells are important examples of polygonal meshes.

Nowadays, a limited use of polygonal meshes is restricted by a small number of accurate discretization schemes. We describe the new mimetic discretizations for a diffusion-reaction problem formulated as a system of two first-order equations. The discretization technique results in a method which is exact for linear solutions. The method is second order accurate for general problems with or without material discontinuities and relatively easy to solve (it produces a symmetric positive definite matrix). The new discretization technique can be extended to polyhedral meshes and some other PDEs. 


\title{
Towards a Variational Complex of the Finite Element Method
}

\author{
Elizabeth L. Mansfield \\ University of Kent, UK
}

\begin{abstract}
Exact differential complexes are important in the design of finite element approximate schemes. This poster starts with these and discusses how they may be extended to a full variational complex. The motivation is to be able to answer the question, "Can you design a finite element scheme for a system which inherits both variational principle and certain pre-selected conservation laws exactly?" This is joint work with Reinout Quispel (Latrobe University, Australia).
\end{abstract}




\title{
Why Mixed Finite Elements are not used in the Petroleum Industry and what can we do about it? (poster)
}

\author{
Ilia D. Mishev \\ Upstream Research Company, Exxon-Mobil
}

\begin{abstract}
The purpose of this poster is to invigorate the dialogue between the academia and the industry. We start with a short description of the most common formulation used in the petroleum industry to model the fluid flow in porous media and discuss what are the implications for the discretizations. One approach based on primal dual Mixed Finite element method will be considered and some examples given.
\end{abstract}




\title{
Asymptotic-Preserving Discretization Schemes
}

\author{
Jim E. Morel \\ Transport Methods Group, CCS-4, Los Alamos National Laboratory
}

\begin{abstract}
Asymptotic limits associated with partial differential equations are limits in which certain nondimensional parameters in an equation are made "small" relative to other nondimensional parameters. The asymptotic solution is generally found to satisfy an equation that is much simpler than the original full equation. When the scale lengths associated with the asymptotic solution are much larger than the smallest scalelengths associated with the full equation, it becomes essential from a numerical point of view to use a discretization scheme for the full equation that "preserves" the asymptotic limit. An asymptoticpreserving scheme is one that yields accurate asymptotic solutions whenever the scale lengths associated with the asymptotic solution are resolved by the mesh. If a scheme is not asymptotic-preserving, accurate asymptotic solutions will be obtained only if the smallest scale lengths associated with the full equation are resolved by the mesh. Because asymptotic scale lengths can be arbitrarily larger than the smallest scale lengths of the full equation, this requirement can make asymptotic calculations prohibitively expensive for discretization schemes that are not asymptotic-preserving. We discuss spatial discretization schemes for the radiation transport equation in the asymptotic diffusion limit. The smallest spatial scale lengths associated with the transport equation are on the order of a mean-free-path (the mean-distance between particle interactions). A truncation error analysis for any consistent transport spatial discretization scheme will indicate that convergence to a smooth solution is guaranteed whenever the spatial cell widths measured in mean-free-paths go to zero. However, the scale length associated with the diffusion limit can be arbitrarily large with respect to a mean-free-path. Thus it can be essential to use asymptotic-preserving discretization schemes in highly diffusive calculations. We show that the simple upwind scheme does not preserve the asymptotic diffusion limit, while a discontinuous Galerkin scheme with a linear trial space does preserve this limit. Both theory and computational examples are presented.
\end{abstract}




\title{
Mimetic Preconditioners for Mixed Discretizations of the Diffusion Equation (poster)
}

\author{
J. David Moulton \\ Los Alamos National Laboratory
}

\begin{abstract}
Joint work with Travis M. Austin, M. Shashkov, and Jim E. Morel.
Mixed discretizations (e.g., mimetic, or compatible) are based on the first order form, and hence, naturally lead to an indefinite linear system. Although optimal preconditioners have been developed for the case of orthogonal grids and a diagonal diffusion tensor, the performance of these methods degrades with full tensor anisotropy or severe grid distortion. Thus, a significant hurdle in the widespread adoption of these discretization methods is the lack of robust and efficient solvers for the corresponding linear system. To this end we are motivated by one specific advantage that the hybrid or local forms of mixed discretizations exhibit, namely, their more localized sparsity structure. Specifically, for the support operator method (SOM) we consider augmentation of the flux (i.e., vector unknowns) such that an appropriate ordering of the augmented flux leads to a new block diagonal system for this component. In contrast to the block diagonal structure of the hybrid system this system has blocks centered about vertices, and block elimination of the flux (i.e., formation of the Schur complement) leads to a symmetric positive definite scalar problem with a standard cell-based 9-point structure (in two dimensions). This reduced system is readily solved with existing robust multigrid methods, such as Dendy's Black Box Multigrid (BoxMG). An analogous approach is used to augment the hybrid or local SOM system and derive the equivalent preconditioner for this case. We demonstrate the effectiveness of this preconditioner for logically rectangular severely distorted grids.
\end{abstract}




\title{
Compatible Discretizations, Covolume Algorithms and Differential Forms
}

\author{
Roy Nicolaides \\ Department of Mathematical Sciences, Carnegie Mellon University
}

\begin{abstract}
Compatible discretizations" have become prominent during the last few years, although they have been under development for at least 15 years. In the finite element setting they are exemplified by "edge" and related elements. Less well known are "mimetic" and "covolume" discretizations which may be considered as generalized finite difference techniques. In most compatible discretizations there are good analogs of exact sequence diagrams, ensuring the existence of analogs of vector identities that are valuable for obtaining good error estimates and reliable numerical results. The covolume approach uses "complementary volumes" to achieve compatible discretizations. The complementary volumes are typically tetrahedra and their corresponding Voronoi polyhedra. Use of these dual meshes is what distinguishes the covolume methodology from other compatible discretizations. This talk will begin with a review of the basic covolume methodology and use it to illustrate the main ideas of compatible discretization, exact sequences of spaces and so forth. Following that it will be shown how the covolume technique can be used to discretize differential forms. There is a remarkable parallel between certain operations on differential forms and the basic constructs appearing in covolume approximations. These will be discussed along with applications to partial differential equations on manifolds.
\end{abstract}




\title{
Compatible Reconstructions of Vectors and their Application to the Navier-Stokes Equations
}

\author{
Blair Perot \\ Department of Engineering \& Computer Science, University of Massachusetts, Amherst
}

\begin{abstract}
Compatible spatial discretizations of partial differential equations invariably deal with vector components as the primary variables. This use of individual vector components (rather than entire vectors) is fundamental and closely tied to algebraic topology and differential forms. However, there are situations where the entire vector is required. The Navier-Stokes equations for fluid dynamics exhibit two such situations. First, the advective term in the equations requires a velocity vector to be defined. Second, the Navier-Stokes equations have certain vector conservation statements (like conservation of momentum, kinetic energy, and circulation) that one would like to construct discrete analogs for.
\end{abstract}

Since vectors are not primary quantities, their reconstruction is a numerical approximation and is not uniquely specified when compatible spatial discretizations are used. A number of different vector reconstruction proposals for both structured and unstructured meshes in 2D and 3D are described. The relationship between these reconstructions is explored along with the resulting conservation properties (when they are known to exist). We develop a unified framework in which vector reconstruction is associated with discrete averaging operators, and where compatible vector reconstructions (with conservation statements) have averaging operators which commute with the discrete differential operators and are closely related to them.

This work has application even when vectors are not explicitly required (such as in electromagnetics, elasticity, and Stokes flow). Discrete Hodge star operators with attractive properties can be constructed from compatible vector reconstruction operators (due to the fact that they commute with the differential operators) even when an explicit vector is never required to solve the equations. 


\title{
Discontinuous Galerkin Methods for Maxwell's Equations in Frequency-Domain (poster)
}

\author{
Ilaria Perugia \\ Dipartimento di Matematica, Universit di Pavia
}

\begin{abstract}
Joint work with Paul Houston and D. Schoetzau.
We present recent work on discontinuous Galerkin (DG) methods for the discretization of the Maxwell equations in frequency-domain, based on employing the interior penalty discretization of the curl-curl operator. Direct and mixed methods will be considered for both low-frequency and high-frequency regimes. The performance of the proposed DG methods for Maxwell problems will be demonstrated on a series of model problems with both smooth and singular analytical solutions. Our theoretical and numerical results indicate that DG methods provide viable alternatives to classical conforming edge element discretization schemes.
\end{abstract}




\title{
High Order Symplectic Integration Methods for Finite Element Solutions to Time Dependent Maxwell Equations (poster)
}

\author{
Robert N. Rieben \\ Institute for Scientific Computing Research, Lawrence Livermore National Laboratory
}

\begin{abstract}
We motivate the use of high order integration methods for compatible finite element solutions of the time dependent Maxwell equations. In particular, we present a symplectic algorithm for the integration of the coupled first order Maxwell equations for computing the time dependent electric and magnetic fields in a mixed finite element approach. Symplectic methods have the benefit of conserving total electromagnetic field energy and are therefore preferred over dissipative methods (such as traditional Runge-Kutta) in applications that require highaccuracy and energy conservation over long periods of time integration. We present a conditionally stable, explicit time integration scheme that is up to 5th order accurate along with some numerical examples which demonstrate the superior performance of high order time integration methods.
\end{abstract}




\title{
Two-Phase Flow Modeling (poster)
}

\author{
Beatrice Riviere \\ Department of Mathematics, University of Pittsburgh
}

\begin{abstract}
This poster presents a high order finite element method that naturally handles unstructured meshes and heterogeneous porous media for solving the incompressible two-phase flow problem. In the proposed algorithm, the primary variables are the wetting phase pressure and saturation. They are approximated by discontinuous polynomials of varying degree. The flexibility of discontinuous Galerkin methods has made these methods competitive for modeling flow and transport problems. Some of the advantages include the high order approximation, the easy implementation on unstructured grids, the robustness of the method for equations with discontinuous coefficients and the local mass conservation property. Numerical simulations are given for homogeneous and heterogeneous porous media.
\end{abstract}




\title{
Compatible Discretizations in Lagrangian/Eulerian Resistive MHD Modeling for Z-pinch Applications (poster)
}

\author{
Allen C. Robinson \\ Sandia National Laboratories
}

\begin{abstract}
We give an overview of the use of compatible discretization techniques used in resistive magnetohydrodynamic (MHD) modeling for Z-pinch simulations at Sandia National Laboratories. Z-pinch MHD physics is dominated by moving material regions whose conductivity properties vary drastically as material passes through melt and plasma regimes. At the same time void regions are modeled as regions of very low conductivity. This challenging physical situation requires a sophisticated modeling approach matched by sufficient computational resources to make progress in physical understanding. An Arbitrary-Lagrangian-Eulerian (ALE) operator split methodology for modeling the MHD equations on unstructured grids is described. An implicit treatment of the magnetic diffusion equation, represented using low order vector edge and face elements, gives solutions free from parasitic transients. A matching algebraic multigrid must also be applied to deal with the large null space of the stiffness matrix. We also discuss the important isssue of constrained transport remapping on unstructured grids and how this can be viewed in the framework of low order face elements. An important issue to be resolved associated with the constrained transport algorithm is the accurate reconstruction of the magnetic flux density field given information on the fluxes on element faces.
\end{abstract}

Sandia is a multiprogram laboratory operated by Sandia Corporation, a Lockheed Martin Company, for the United States Department of Energy's National Nuclear Security Administration under contract DE-AC04-94AL85000. 


\title{
Relationships Among Some Locally Conservative Discretization Methods Which Handle Discontinuous Anisotropic Coefficients on Deformed Grids
}

\author{
Thomas F. Russell \\ Division of Mathematical Sciences, National Science Foundation
}

\begin{abstract}
This talk presents the relationships between some numerical methods suitable for a heterogeneous elliptic equation of the form $-\operatorname{div}(K(x) \operatorname{grad} p)=q$, motivated by applications to subsurface flow (pressure or potential) equations. The methods discussed are the classical Raviart-Thomas mixed finite element method (MFEM), the control-volume mixed finite element method (CVMFEM), the support operators method (SOM), the enhanced cell-centered finite difference method (ECCFDM), and the multi-point flux-approximation (MPFA) control volume method. These methods are all locally mass conservative, and handle general irregular grids with anisotropic and heterogeneous discontinuous conductivity $\mathrm{K}(\mathrm{x})$. In addition to this, the methods have in common a weak continuity in the pressure across the edges, which in some cases corresponds to Lagrange multipliers. This weak continuity appears to be an essential property for the accuracy of these methods.

While the methods are applicable in two and three dimensions, the details of the above relationships are presented for logically rectangular quadrilateral grids in 2D. Issues of deformed grids are substantially more complex in 3D. An example (Naff, Russell, and Wilson, Computational Geosciences, 2002) is presented in which the Piola-transformed lowest-order Raviart-Thomas spaces fail to contain the constant-velocity vector fields for a hexahedron that is a trilinear image of a reference cube.
\end{abstract}

This represents joint work with R.A. Klausen and R. Winther of the University of Oslo, Norway. 


\title{
Consistent Material Operators for Geometrical Discretization Methods on Generalized Grids (poster)
}

\author{
Rolf Schuhmann \\ Technische Universität Darmstadt, Institut für Theorie Elektromagnetischer Felder (TEMF)
}

\begin{abstract}
Joint work with Marco Cinalli and Thomas Weiland.
Geometrical methods for the spatial discretization of Maxwell's equation are able to preserve important properties, like the conservation of charge and energy and the orthogonality of solution spaces. The Finite Integration Technique (FIT) provides for a natural and efficient notation of such approaches, introducing separate matrix operators for (exact) topological and (approximate) material relations. The implementation of the material operators strongly depends on the type of computational grids, but must generally fulfill important consistency and stability properties. In this paper we investigate the accuracy and efficiency of some recently developed material operators for tetrahedral grids. Additionally it will be shown that the requirement for consistency leads to some surprising consequences for the overall simulation scheme.
\end{abstract}




\title{
Mimetic Finite Difference Methods for Partial Differential Equations and Discrete Vector and Tensor Analysis
}

\author{
Mikhail Shashkov \\ Theoretical Division, Los Alamos National Laboratory
}

\begin{abstract}
In past 10 years we have developed new high-quality, mimetic finite-difference methods based on discrete analog of vector and tensor analysis (DVTA). The basis of DVTA is the design of discrete operators that preserve certain essential properties of, and relationships between, the corresponding analytic operators. The DVTA is the basis for new techniques for large-scale numerical simulations approximating the solution of partial differential equations (PDEs). The new methods provide a significant extension of the well known and useful finite volume methods and are designed to more faithfully represent important properties of physical processes and the continuum mathematical models of such processes. Algorithms based on these techniques are used for modeling high-speed flows, porous media flows, diffusion processes, and electromagnetic problems. In this presentation we will describe DVTA and demonstrate how it can be used to construct high-quality finite-difference methods for PDEs.
\end{abstract}




\title{
Finite Volume Element Methods for Parabolic Integro-Differential Equation with Nonsmooth Initial Data (poster)
}

\author{
Rajen Kumar Sinha \\ Institute for Scientific Computation, Texas A\&M University
}

\begin{abstract}
Joint work with R. D. Lazarov and R. E. Ewing.
Mathematical models describing the nonlocal reactive flows in porous media and heat conduction through materials with memory give rise to parabolic integro-differential equation. We present a semidiscrete finite volume element (FVE) approximations to parabolic integro differential equation (PIDE) in a two-dimensional convex polygonal domain. More precisely, for homogeneous equation, an elementary energy technique is used to derive optimal error estimate in $L^{2}$ and $H^{1}$ norms for positive time when the given initial function is in $H_{0}^{1}$.
\end{abstract}




\title{
Entropy Stability Theory For Difference Approximations of Quasilinear Problems
}

\author{
Eitan Tadmor \\ Center for Scientific Computation And Mathematical Modeling, University of Maryland, College \\ Park
}

\begin{abstract}
We provide a general overview on the entropy stability of difference approximations in the context of quasilinear conservation laws, and related time-dependent problems governed by additional dissipative and dispersive forcing terms.

As our main tool we use a comparison principle, comparing the entropy production of a given scheme against properly chosen entropy-conservative schemes. To this end, we introduce closed-form expressions for new (families) of new entropy-conservative schemes, keeping the "perfect differencing" of the underlying differential form. In particular, entropy stability is enforced on rarefactions while keeping sharp resolution of shock discontinuities.

A comparison with the numerical viscosities associated with these entropy-conservative schemes provides a useful framework for the construction and analysis of existing and new entropy stable scheme. We employ this framework for a host of first- and second-order accurate schemes. The comparison approach yields precise characterizations of entropy stable semi-discrete schemes for both scalar problems and multi-dimensional systems of equations. We extend these results to the fully discrete case, where the question of stability is settled under optimal CFL conditions using a complementary approach based on homotopy arguments.
\end{abstract}




\title{
Finite Element Methods for Non-elliptic but Coercive Problems (poster)
}

\author{
Jean-Marie Thomas \\ Applied Mathematics, University of Pau, France
}

\begin{abstract}
One analyze finite element methods for a variational problem : find $u \in V$ such that $a(u, v)-k(u, v)=l(v)$ for any $v \in V$, where $(\cdot, \cdot)$ is the scalar product of an Hilbert space $H, V$ is an Hilbert space with continuous imbedding of $V$ into $H$ and $V$ is dense in $H, a(\cdot, \cdot)$ is a symmetric non-negative continuous bilinear form on $V \times H$ such that $a(\cdot, \cdot)+(\cdot, \cdot)$ is $V$-elliptic. Moreover we assume that $k$ is not an eigenvalue of the associated spectral problem. By the finite element method, the discrete problem associated to a finite dimensional subspace of $V$ consists to find $u_{h}$ in $V_{h}$ such that $a\left(u_{h}, v_{h}\right)-k\left(u_{h}, v_{h}\right)=l\left(v_{h}\right)$ for any $v_{h} \in V_{h}$. One looks for an optimal a priori error bound similar to Céa's Lemma for elliptic problems. In some situations, this result is obtained as soon as $h$ is sufficiently small. In the other situations, we show what additional property must verify $V_{h}$ for obtaining again the optimal a priori error bound. Applications to the analysis of some time-harmonic systems from elastodynamics, aeroacoustics and electromagnetism will be presented. At last, primal and dual finite element approximations of the Helmholtz equation will be considered.
\end{abstract}




\title{
Formal Theory of PDEs and simulation of Fluid Flows \\ (poster)
}

\author{
Jukka Tuomela \\ Department of Mathematics, University of Joensuu
}

\begin{abstract}
Joint work with Bijan Mohammadi.
In many physical models there appear constraints or conserved quantities which make the system essentially overdetermined. We propose a ne $\mathrm{w}$ approach to solve numerically these kind of systems of PDEs which is based on the formal theory of PDEs. The idea is to find the involut ive form of the system, and use it explicitly in the computations. The involutive form is important because many properties of the system cannot be determined if the system is not involutive. We illustrate our approach by considering a compressible flow problem.
\end{abstract}




\title{
Coupling of MFE or Mimetic Finite Differences with Discontinuous Galerkin for Poro-Elasticity
}

\author{
Mary Fanett Wheeler \\ Institute for Computational Engineering and Sciences, The University of Texas at Austin
}

\begin{abstract}
In this presentation we discuss the modeling of Biot's consolidation model for poro-elasticity in porous media. Here we employ a mixed finite element or a mimetic finite difference method for approximating the pore pressure and a discontinuous Galerkin method for approximating displacements. This approach yields a locally conservative flow field and continuous numerical fluxes and forces. Moreover numerical eperiments indicate that the pore pressure is non-oscillatory even in low permeability regions. Theoretical error estimates and computational results will be presented to demonstrate this scheme.
\end{abstract}




\title{
Development and Application of Compatible Discretizations of Maxwell's Equations
}

\author{
Daniel A. White \\ Center for Applied Scientific Computing, Lawrence Livermore National Laboratory
}

\begin{abstract}
This talk will be an overview of a variety of electromagnetics problems that are of interest to LLNL, and of the numerical methods that have been developed to solve these problems. We will briefly discuss the history of Finite Difference Time Domain methods Discrete Surface Integral methods, and Discrete Differential Form based Finite Element Methods. Our current research efforts are in the area of higher-order finite elements, higher-order time integration, and hybrid finite element boundary element simulations, and we will present recent results in these areas. Applications that we are applying these algorithms to include: photonic bandgap structures, linear accelerators, wave propagation in random environments, and microwave circuits.
\end{abstract}

This represents joint work with Joe Koning and Rob Rieben. 


\title{
From the de Rham Sequence to Mixed Elasticity
}

\author{
Ragnar Winther \\ Centre of Mathematic for Applications, University of Oslo, Norway
}

\begin{abstract}
There have been many attempts during the past four decades to construct stable mixed finite elements for the Hellinger-Reissner formulation of linear elasticity, i.e., the stress-displacement formulation. Unfortunately, these efforts have not been as successful as expected. There is now renewed interest in this topic due to applications of mixed models in areas such as viscoelasticity, where the stress-strain relation may be nonlocal, and, as a consequence, a pure displacement model is excluded. In this talk, we first explain why the condition of symmetry of the stress tensor makes it difficult to construct stable, low order, mixed finite elements for elasticity. By introducing a proper commuting diagram, we establish a connection between the standard de Rham sequence and a corresponding "elasticity sequence." Utilizing discrete versions of this connection, we are then able to construct new stable elements in two and three space dimensions, which satisfy either the usual symmetry condition or a weak version of this condition.
\end{abstract}

This represents joint work with Douglas N. Arnold, Univ. of Minnesota and Richard S. Falk, Rutgers University. 



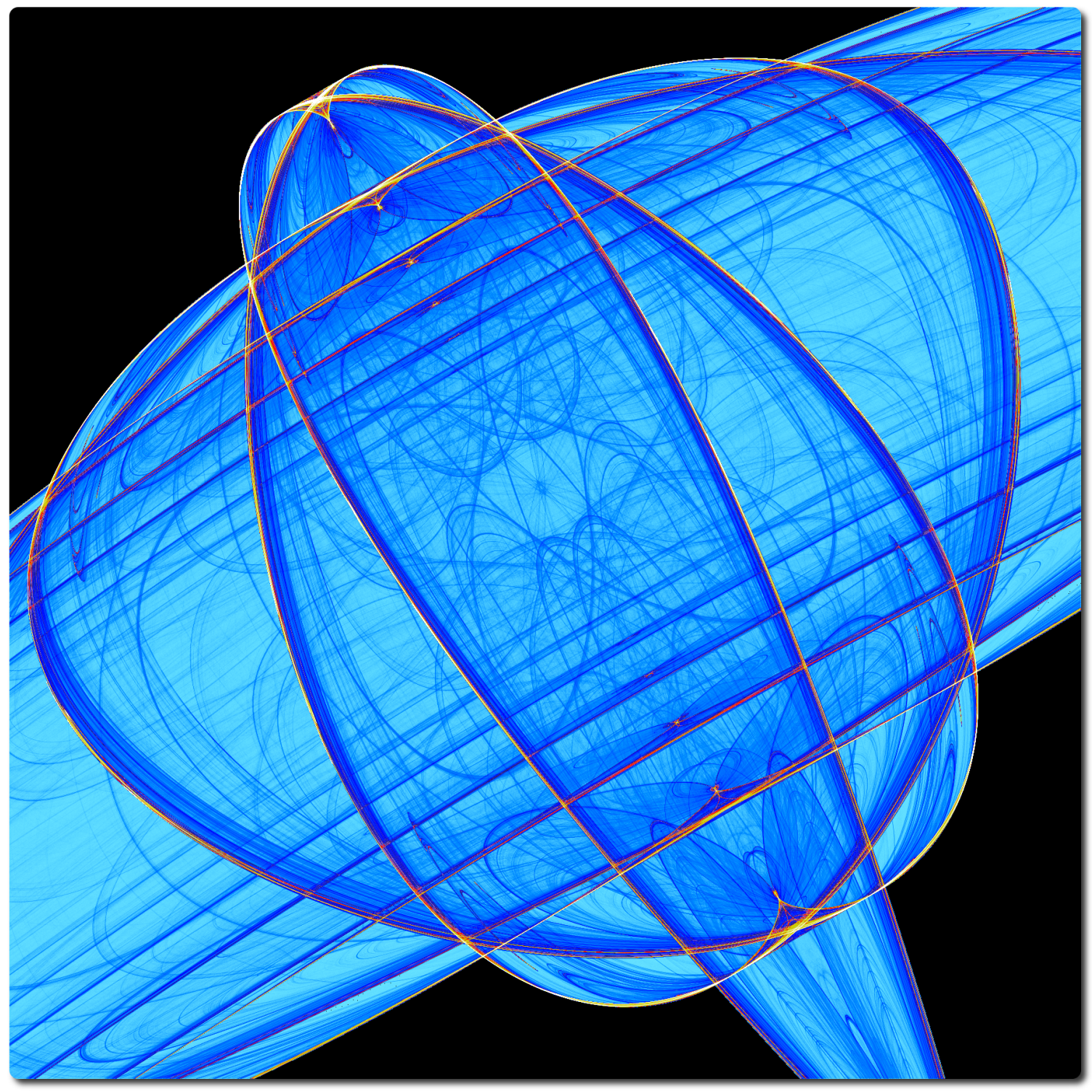

\section{www.ima.umn.edu}

IMA Participating Institutions: Consiglio Nazionale delle Ricerche, Georgia Institute of Technology, Indiana University, Iowa State University, Kent State University, Lawrence Livermore National Laboratory, Los Alamos National Laboratory, Michigan State University, Mississippi State University, Northern Illinois University, Ohio State University, Pennsylvania State University, Purdue University, Rice University, Sandia National Laboratories, Seoul National University (BK21 Math-SNU), Seoul National University (SRCCS), Texas A\&M University, University of Chicago, University of Cincinnati, University of Delaware, University of Houston, University of Illinois, University of Iowa, University of Kentucky, University of Maryland, University of Michigan, University of Minnesota, University of Notre Dame, University of Pittsburgh, University of Texas at Austin, University of Wisconsin, University of Wyoming, Wayne State University

IMA Participating Corporations: Boeing, Corning, ExxonMobil, Ford, General Electric, General Motors, Honeywell, IBM, Johnson \& Johnson, Lockheed Martin, Lucent, Motorola, Schlumberger, Siemens, Telcordia Technologies, 3M 